positive $\geq 15 \mathrm{~mm}$ for those with prior BCG. Children with borderline or positive Mantoux test results, or in whom there was clinical concern, were referred for consultant assessment and/or IGRA (Quantiferon Gold). Data were collected retrospectively from case notes.

Results 976 children were referred. 756 completed initial assessment (388 (51\%) male, mean age $6.2 \pm 4.6$ years, range $0.16-16.36$ years). BCG history was known in 754 (99.7\%; 516 BCG). 403 patients were discharged without intervention, 63 were offered BCG vaccination, two were referred elsewhere and 288 were referred for consultant assessment. Of these 288, 108 were notified with TB, 46 received chemoprophylaxis, 117 received no treatment, 5 received BCG and 12 failed to attend. 252 children had paired Mantoux and IGRA. Of these, 18/44 (41\%) of those with a borderline Mantoux had a positive IGRA. 126/252 had TB infection (91 active and 35 latent TB)-see Abstract P175 table 1. A Mantoux threshold of $\geq 15 \mathrm{~mm}$ identified 77 (61\%) children with TB infection, IGRA identified 92 (73\%) and the two tests combined identified 100 (79\%) children.

Abstract P175 Table 1 Mantoux and IGRA in children with TB infection

\begin{tabular}{lclcr}
\hline & GIFN negative & GIFN indeterminate & GIFN positive & Total \\
\hline Mantoux neg & 18 & 1 & 5 & 24 \\
Mantoux borderline & 6 & 1 & 18 & 25 \\
Mantoux positive & 5 & 3 & 69 & 77 \\
Total & 29 & 5 & 92 & 126 \\
\hline
\end{tabular}

Conclusion Using a Mantoux threshold of $\geq 15 \mathrm{~mm}$ induration significantly underestimates the number of children with TB infection compared with using Mantoux and IGRA together.

\section{P176 DOES BCG PROTECT AGAINST ATOPIC DISEASES ONLY FOR A LIMITED TIME PERIOD?}

doi:10.1136/thoraxjnl-2011-201054c.176

M F Linehan, R M Niven, D N Baxter, T L Frank. The University of Manchester, Manchester, UK

Allergic diseases such as atopic asthma are believed to have their origins in early life $e^{1}$ but the precise mechanisms and timings of the relevant immunoregulation continue to be a focus of research. One area of investigation has been the role of vaccinations in Th cytokine regulation. ${ }^{1}$ Epidemiological studies investigating the potential of BCG, a potent immunomodulator, to reduce the risk of atopic diseases report conflicting results. ${ }^{2}$ A Manchester study (MANCAS), using a cohort of children all born in the same hospital in the mid 1990s identified a lower risk of wheeze for children given neonatal BCG. ${ }^{3}$ Data analysis for a follow-up study 6 years later when the cohort was aged 13-17 yrs has just been completed. Using the same definitions for wheeze and asthma as the first study there was no difference in prevalence of wheeze or asthma between BCG vaccinated and non-vaccinated adolescents (Abstract P176 table 1). Significance tests between the studies were not performed because some participants responded only to one of the two studies. A Medical Research Council study ${ }^{4}$ in the 1950s investigating the effectiveness of a vaccination programme to prevent tuberculosis identified a progressive decrease in the efficacy of BCG in successive 5 year periods with $80 \%$ efficacy 5 years post vaccination reducing to $59 \% 10-15$ yrs after vaccination. If the decrease in efficacy of BCG modifies its ability to protect against atopy it may be that the conflicting results in studies investigating BCG and atopy have occurred because the protection afforded by BCG is limited to within a timeframe after vaccination.
Abstract P176 Table 1 Asthma/wheeze prevalence

\begin{tabular}{ccll}
\hline & Asthma (3K0) & Asthma (3K01MoS) & Wheeze \\
\hline MANCAS1 $n=2414 / 5086$ & & \\
NN BCG & $21.6 \%(201)$ & $13.6 \%(126)$ & $17.2 \%(168)$ \\
No BCG & $26.2 \%(266)$ & $17.5 \%(176)$ & $23.2 \%(250)$ \\
& $\mathrm{p}=0.02$ & $\mathrm{p}=0.02$ & $\mathrm{p}<0.01$ \\
MANCAS2 $\mathrm{n}=1608 / 6338$ & & $15.9 \%(112)$ \\
NN BCG & $17.0 \%(123)$ & $10.7 \%(77)$ & $14.4 \%(103)$ \\
No BCG & $16.3 \%(120)$ & $11.4 \%(84)$ & $\mathrm{p}=0.44$ \\
\hline
\end{tabular}

\section{REFERENCES}

1. Marchant, et al. T cell-mediated immune responses in human newborns: ready to learn? Clin Exp Immunol 2005;141:10-18.

2. Arnoldussen, et al. BCG vaccination and allergy: a systematic review and metaanalysis. JACl 2011;127:246-53.

3. Linehan, et al. Is the prevalence of wheeze in children altered by neonatal BCG vaccination? JACl 2007;119:1079-85.

4. Medical Research Council. BCG and vole bacillus vaccines in the prevention of tuberculosis in adolescence and early adult life. Bull WHO 1972;46:371-85.

\section{P177 EVOLUTION OF LUNG FUNCTION IN PRIMARY CILIARY DYSKINESIA: A TWO CENTRE RETROSPECTIVE STUDY}

doi:10.1136/thoraxjnl-2011-201054c.177

${ }^{1} \mathrm{M}$ Maglione, ${ }^{2} \mathrm{~A}$ Bush, ${ }^{2} \mathrm{C}$ Hogg, ${ }^{1} \mathrm{~S}$ Montella, ${ }^{1} \mathrm{~F}$ Santamaria. ${ }^{1}$ Department of Paediatrics, Federico II University, Naples, Italy; ${ }^{2}$ Department of Respiratory Medicine, Royal Brompton Hospital, London, UK

Background Evolution of spirometry in primary ciliary dyskinesia (PCD) and its determinants are unclear. To assess morbidity and burden of this condition, we investigated the progression of spirometry in patients from two European centres.

Methods Ninety-six patients with $=1$ spirometry (Naples, Italy, $\mathrm{n}=21$; London, UK, $\mathrm{n}=75$ ) were enrolled. Sixty-five (Naples, $\mathrm{n}=15$; London, $\mathrm{n}=50$ ) with 4 years spirometry were analysed longitudinally. Best annual $\mathrm{FEV}_{1}$, corresponding FVC, both expressed as z-scores, and sputum culture results were recorded.

Results In Naples and London, age at referral to the centre was 9.8 (range, 0.1-20.2) and 6.1 years (range, 0.1-17.3), respectively $(\mathrm{p}=0.02)$, while age at first spirometry was 11.6 (range, 8.1-20.2) and 8.4 years (range, $4.2-17.3)$, respectively $(p<0.001)$. In both centres patients with situs in versus (Naples, $n=15$; London, $n=36$ ) were referred earlier $(p<0.001)$. Despite later diagnosis, Naples children had better baseline $\mathrm{FEV}_{1}$ and FVC z-scores $(-0.53$ (1.60) vs -1.66 (1.35), and $0.50(1.55)$ vs $-1.36(1.32), p<0.001$ respectively) when first seen. Slopes of $\mathrm{FEV}_{1} \mathrm{z}$-scores over 4 years were -0.05 (95\% CI -0.36 to 0.26 ) and 0.05 (95\% CI -0.05 to 1.65$)$ in Naples and London, respectively $(p=0.38)$. No significant correlation was found between slopes of $\mathrm{FEV}_{1}$ z-scores and age at referral or baseline $\mathrm{FEV}_{1} \mathrm{z}$-score. Haemophilus influenzae was the most frequently isolated pathogen ( $95 \%$ and $79 \%$ of subjects in Naples and London, respectively, $\mathrm{p}=0.1$ ). Naples subjects had higher prevalence of $P$ seudomonas aeruginosa $(62 \%$ vs $36 \%, p=0.04)$. Paeruginosa isolation was not associated with worse baseline $\mathrm{FEV}_{1}$ z-scores or slopes of $\mathrm{FEV}_{1}$ z-scores.

Conclusions The better lung function despite later diagnosis in Naples is apparently unexplained. However, as spirometry in PCD is stabilised during treatment, its short-term evolution is not related to age at referral or to baseline $\mathrm{FEV}_{1}$. Spirometry is thus not a useful end-point for randomised controlled trials of treatment. Late diagnosis is common for patients without situs anomalies. Although the potential impact of $P$ aeruginosa infection on PCD lung function is unclear, its unexpectedly high prevalence merits further study to determine best prevention and management strategies. 